\title{
Criterios programáticos para la innovación docente en ciencias de la empresa: planificación didáctica orientada a la realidad empresarial
}

\author{
Rey-García, Marta'; Salido-Andrés, Noelia ${ }^{2}$ \\ ${ }^{1}$ Universidade da Coruña, Facultade de Economía y Empresa, 0000-0002-9453-8551 \\ ${ }^{2}$ Universidade da Coruña, Facultade de Economía y Empresa, 0000-0002-9692-1650
}

\section{RESUMEN}

Conseguir un aprendizaje efectivo en el campo de las ciencias de la empresa requiere de innovación docente continua y orientada a un triple esfuerzo de integración. En primer lugar, el diseño del plan didáctico de las materias debe integrar contenidos pertenecientes a distintos niveles de análisis. Desplegarlos con eficacia dentro y fuera del aula requiere, en segundo lugar, de una continua transición entre recursos relativos a los fundamentos teórico-conceptuales de las materias y a los problemas prácticos de la empresa en un entorno de cambio acelerado; de modo que los alumnos visibilicen y contextualicen los retos a los que se enfrentan en la realidad, su relevancia y las posibles soluciones. En tercer lugar, es aconsejable combinar una pléyade de metodologías activas que impacten tanto sobre la mejora de sus competencias como de su empleabilidad. El objetivo del presente trabajo es fundamentar metodológicamente el diseño e implementación de un plan didáctico innovador para materias de empresa acorde a los tres criterios programáticos señalados. Se utilizará como caso de estudio el desarrollo del plan didáctico de la materia 'Distribución Comercial' (2011-2019), con especial énfasis en su articulación metodológica a través del aprendizaje colaborativo por proyectos. Por último, se valorará la aplicabilidad de esta experiencia innovadora a otras materias del ámbito de las ciencias de la empresa.

PALABRAS CLAVE: Plan didáctico innovador, metodologías activas, aprendizaje colaborativo por proyectos, ciencias de la empresa, sostenibilidad empresarial 


\section{CITA RECOMENDADA:}

Rey-García, Marta; Salido-Andrés, Noelia (2020): Criterios programáticos para la innovación docente en ciencias de la empresa: planificación didáctica orientada a la realidad empresarial. En De la Torre Fernández, E. (ed.) (2020). Contextos universitarios transformadores: Boas prácticas no marco dos GID. IV Xornadas de Innovación Docente. Cufie. Universidade da Coruña. A Coruña (págs. 207-222). DOI capítulo: https://doi.org/10.17979/spudc.9788497497756.207 DOl libro: https://doi.org/10.17979/spudc. 9788497497756

\section{ABSTRACT}

Achieving effective learning in the field of Business Studies requires continuous teaching innovation aimed at a three-fold integration effort. Firstly, the design of the teaching plan must integrate contents related to multiple levels of analysis. Secondly, deploying them within and outside the classroom requires a continuous transition between resources relative to the theoretical-conceptual contents and to the practical problems posed to businesses by an environment in accelerated change; so that students visualize and contextualize the real challenges being faced, their relevance and the potential solutions. Thirdly, a plethora of active learning methodologies should be combined in order to improve not only students' competencies but also their employability. In this context, the objective of this paper is to methodologically substantiate the design and implementation of an innovative teaching plan in the field of Business Studies in accordance with the three above-mentioned programmatic criteria. A case study of its development for the 'Retail Management' course (2011-2019) will be presented, with special emphasis on its methodological approach through collaborative project-based learning. Finally, the applicability of this teaching innovation experience for other subjects in the Business Studies field will be assessed.

KEY WORDS: Innovative teaching plan, active learning, collaborative project-based learning, business studies, corporate sustainability 


\section{INTRODUCCIÓN}

El plan didáctico es el documento que orienta el estudio y conecta el material didáctico a los procesos cognitivos del alumno para que este pueda trabajarlo tanto autónomamente como dentro del aula. Es un elemento motivador clave que debe despertar el interés por la materia, guiar y facilitar el aprendizaje, ayudar a comprender y aplicar los diferentes conocimientos, e integrar los medios y recursos de apoyo para el aprendizaje (García, 2014). Se trata, en definitiva, del "andamiaje preciso para el logro de competencias" (García, 2014, p. 2).

Conseguir un aprendizaje efectivo en un campo tan dinámico como el de las ciencias de la empresa requiere de innovación continua en lo que al diseño, ejecución y evaluación del plan didáctico se refiere. En este contexto, el objetivo del presente trabajo es fundamentar metodológicamente el diseño e implementación de un plan didáctico innovador para las materias de empresa acorde a un triple criterio programático: 1) integración de niveles de análisis; 2) integración de contenidos teórico-prácticos; y 3) integración de metodologías activas.

Para ello, se utilizará como caso de estudio el desarrollo en la Universidad de A Coruña de la materia Distribución Comercial/Retail Management, perteneciente al Área de Comercialización e Investigación de Mercados, e impartida en las titulaciones de ADE y ADE y Derecho durante el periodo 2011-2019. Por último, se valorará la aplicabilidad de esta experiencia de innovación docente para otras materias del ámbito de las ciencias de la gestión empresarial.

\section{UNA PROPUESTA DE CRITERIOS PROGRAMÁTICOS PARA LA INNOVACIÓN DOCENTE EN LAS CIENCIAS DE LA EMPRESA}

La propuesta de criterios programáticos está orientada a guiar la innovación docente en las ciencias de la empresa a través de un triple esfuerzo de integración. En primer lugar, exige que el docente responsable de la elaboración del plan didáctico de las materias integre contenidos pertenecientes a tres niveles de análisis: 1) un nivel macro donde se dan cita las grandes tendencias del entorno que impactan sobre las estrategias empresariales; 2) un nivel meso 
relativo al desempeño de las organizaciones, empresariales o no, que operan en los mercados; y 3) un nivel micro relativo a las preferencias, percepciones y comportamientos de los consumidores, prospectos 0 trabajadores, entre otros actores individuales de relevancia. En segundo lugar, desplegar con éxito ese diseño tanto dentro como fuera del aula requiere de iterar la transición desde recursos relativos a contenidos teórico-conceptuales que fundamentan la materia, hacia aquellos que atañen a los retos prácticos de una realidad empresarial en cambio acelerado (aproximación deductiva); y viceversa (aproximación inductiva). Por último, implementar esta planificación de contenidos y recursos exige combinar una pléyade de metodologías activas, tanto dentro como fuera del aula, entre las que cabría mencionar el aprendizaje por proyectos o project-based learning, el aprendizaje colaborativo, el aprendizaje basado en problemas o problem-based learning, el método del caso, el role play 0 los experimentos en el aula. Estas metodologías activas impactan positivamente tanto sobre el proceso de aprendizaje por competencias como sobre la empleabilidad de los estudiantes (Figura 1).

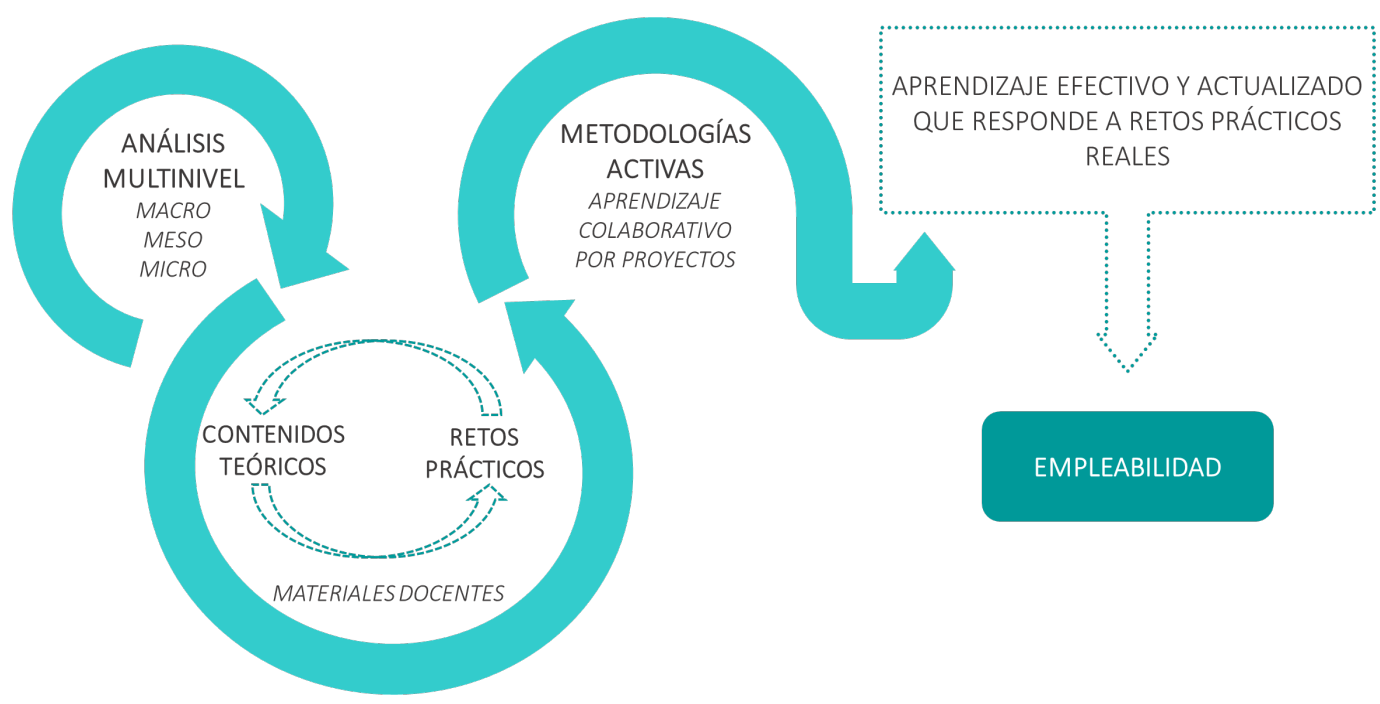

Fuente: elaboración por las autoras.

Figura 1. Propuesta programática para un plan didáctico innovador en ciencias de la empresa. 


\subsection{INTEGRACIÓN DE NIVELES DE ANÁLISIS}

Para comprender el entorno que rodea a las empresas, los alumnos han de desarrollar una visión sistémica que les permita desentrañar las complejas interacciones entre distintos niveles de análisis de la realidad. El nivel macro 0 de análisis del entorno se aborda frecuentemente mediante alguna variante del análisis PESTEL (Political, Economic, Social, Technological, Environmental y Legal), utilizado para identificar los factores del entorno que pueden favorecer 0 amenazar la posición competitiva de manera determinante (SammutBonnici \& Galea, 2015). Aplicada a la docencia permite a los alumnos explorar las tendencias que impactan sobre la gestión empresarial más allá del ámbito estrictamente económico.

En cuanto al nivel meso, el éxito empresarial radica, en el medio y largo plazo, en la capacidad de la organización para innovar en su interacción con los proveedores, los clientes y otros grupos de interés relevantes, de manera que las relaciones planteadas generen valor compartido -económico, social y medioambiental- para todas las partes implicadas 0 stakeholders, siempre desde la plena integración en la filosofía general de la empresa de orientación a mercado y el alineamiento con los modelos de negocio.

En cuanto al nivel micro, el factor dinámico más destacado es el empoderamiento del consumidor transformado digitalmente. La transformación digital ha revolucionado el viaje del cliente (customer journey) y alimentado sus expectativas de omnicanalidad: los nuevos consumidores digitales esperan experiencias de cliente consistentes en todo momento, desde cualquier lugar y a través del canal que eligen para interactuar con las empresas.

\subsection{INTEGRACIÓN DE CONTENIDOS Y RECURSOS TEÓRICOS Y PRÁCTICOS}

Por si integrar de forma eficaz estos tres niveles de análisis no fuese reto de entidad suficiente, desplegarlos dentro y fuera del aula requiere integrar recursos que faciliten una continua transición entre los contenidos teórico-conceptuales que fundamentan la materia y los retos prácticos empresariales. La combinación de sesiones basadas en la lección magistral con sesiones de carácter interactivo o tutorías en grupo reducido es condición necesaria, pero no 
suficiente, para iterar con éxito esa transición de la teoría a la práctica y de la práctica a la teoría. Para conseguir una verdadera integración en cuanto a contenidos hay que innovar de manera continua los recursos pedagógicos y materiales docentes.

En ausencia de manuales actualizados o casos de estudio ad hoc, la grey literature constituye una fuente de especial potencial: documentos producidos por think tanks, organizaciones gubernamentales y no gubernamentales, consultoras 0 asociaciones sectoriales. Ahora bien, deberán cumplir dos requisitos: 1) serán filtrados y valorados por el profesor universitario en cuanto a unos mínimos niveles de rigor metodológico, poniendo de manifiesto sus limitaciones metodológicas, si las hubiere, ante el alumnado; y 2) serán incardinados de los contenidos teóricos correspondientes en el devenir del plan didáctico, de modo que el alumno sepa en todo momento y de manera concreta qué conceptos y qué relaciones entre conceptos se pretende ilustrar con casos reales; y aprenda a conectarlos de forma deductiva 0 inductiva.

\subsection{INTEGRACIÓN DE METODOLOGÍAS ACTIVAS}

En tercer y último lugar, culminar la implementación de un plan didáctico diseñado acorde a los dos anteriores criterios programáticos aconseja combinar una pléyade de metodologías activas, tanto dentro como fuera del aula. Las metodologías activas están dirigidas a mejorar no solo la interactividad entre profesor y alumnos y el aprendizaje individual o colaborativo de Ios propios alumnos, sino también a reforzar la conectividad entre el aprendizaje universitario y la resolución de retos de organizaciones reales en el entorno socioeconómico del alumnado; impactando en último término sobre la mejora de su empleabilidad.

Entre las metodologías activas al servicio de la docencia orientada a la resolución de retos de la práctica empresarial destacan el aprendizaje colaborativo y el aprendizaje por proyectos 0 project-based learning. La primera consiste en una actividad estructurada que implica activamente a los estudiantes en la resolución colaborativa de problemas comunes, convirtiéndolos en responsables tanto del aprendizaje propio como conjunto (Barkley et al. 2012). En cuanto al aprendizaje por proyectos, este se produce cuando como resultado del 
proceso de aprendizaje se obtiene un proyecto en base al cual se articula la actividad formativa (Fernández, 2006). Empleadas de forma conjunta, estas metodologías son adecuadas para optimizar el aprendizaje académico con la mejora de la empleabilidad de los estudiantes, muy especialmente en asignaturas interdisciplinares, al preparar y capacitar a los estudiantes para la construcción de su inminente carrera profesional en contextos laborales complejos, cambiantes y diversos, favoreciendo el desarrollo de competencias transversales y habilidades interpersonales altamente demandadas en el mercado laboral actual como la predisposición favorable al autoaprendizaje, el pensamiento diferente 0 creativo, el trabajo organizado, el desempeño competitivo, o la colaboración orientada a objetivos (Barkley et al. 2012; Espinosa et al. 2006; Fernández, 2006).

\section{DISEÑO E IMPLEMENTACIÓN DE UN PLAN DIDÁCTICO INNOVADOR EN EL ÁREA DE COMERCIALIZACIÓN E INVESTIGACIÓN DE MERCADOS: EL CASO DE LA MATERIA "DISTRIBUCIÓN COMERCIAL / RETAIL MANAGEMENT"}

"Distribución Comercial/Retail Management" (Código: 611G02030) es una asignatura obligatoria con valor de 6 créditos que se oferta desde el Área de Comercialización e Investigación de Mercados de la Universidad de A Coruña (UDC). Esta materia se ofrece dentro del segundo cuatrimestre de dos titulaciones de Grado desde el curso académico 2011/2012: el tercer curso del Grado en Administración y Dirección de Empresas (GADE) (tanto en castellano/gallego como inglés), y el quinto curso del doble Grado en Administración y Dirección de Empresas y Derecho (GADE+DER). Los objetivos, contenidos y sistema de evaluación se encuentran recogidos en términos generales en su guía docente (Rey-García, 2011-2019).

El objetivo general de la asignatura consiste en introducir a los estudiantes en la realidad del sector de distribución comercial en general y de la distribución minorista en particular, haciendo hincapié en la gestión estratégica de esta área del marketing empresarial y en las oportunidades que supone para la mejora competitiva de las organizaciones y para la 
satisfacción de sus clientes. La asignatura cuenta con un doble valor diferencial. Por un lado y debido al momento en que se imparte en ambos planes de estudios- a los alumnos que la cursan se les presupone un conocimiento intermedio de la disciplina del marketing, mediado por la cercanía o inminencia de su graduación y por su lógica preocupación acerca de su futuro laboral y de su empleabilidad. Por otro lado, dado que se trata de una asignatura paradigmática del fuerte dinamismo y orientación práctica que caracterizan a la disciplina del marketing, requiere integrar la adquisición de conocimientos teóricos con su aplicación a interacciones reales entre organizaciones e individuos participantes en el sector de la distribución; y también integrar contenidos relativos a niveles de análisis institucional, empresarial y de comportamiento del consumidor (Rey-García, 2019b).

El diseño del plan didáctico, además de orientarse al aprendizaje de conocimientos específicos de la distribución comercial, incide sobre la adquisición de competencias transversales en el ámbito de la gestión empresarial, como la comunicación persuasiva, la negociación o el trabajo en equipo, facilitando un aprendizaje por competencias comerciales que influye sobre la mejora de la empleabilidad del alumnado. A continuación se detalla el modo en que se ha diseñado e implementado dicho plan acorde a los tres criterios programáticos aquí propuestos para el periodo 2011-2019.

\subsection{INTEGRACIÓN DE NIVELES DE ANÁLISIS: LA DISTRIBUCIÓN COMO SECTOR, INSTITUCIÓN E INTERCAMBIO}

El docente responsable de la elaboración del plan didáctico ha integrado contenidos pertenecientes a los distintos niveles de análisis con el objetivo de que los estudiantes comprendan que las decisiones de distribución que se tomen respecto a cada uno de ellos son interdependientes y que han de estar perfectamente coordinadas para poder establecer y mantener relaciones de intercambio satisfactorias. En concreto:

- A nivel macro se integra la discusión de las tendencias específicas del sector de la distribución comercial (i.e. plataformización, internacionalización, colaboración, 
concentración, customización, desintermediación, etc.), con la de los factores del entorno que impactan transversalmente sobre las estrategias de distribución; en especial la transformación digital (comercio online y omnicanalidad) y la sostenibilidad de la cadena de suministro de las empresas de distribución.

- A nivel meso, las funciones y dinámicas de interacción de las distintas organizaciones que participan en el canal ocupan un lugar central, en el entendimiento de que la distribución comercial es una función empresarial estratégica.

- A nivel micro, se abordan las microdecisiones en materia de surtido, merchandising 0 logística en el punto de venta -físico o virtual-; así como todo lo relativo al impacto de la distribución sobre el viaje del consumidor individual y su experiencia de cliente.

\subsection{INTEGRACIÓN DE CONTENIDOS Y RECURSOS TEÓRICOS Y PRÁCTICOS: PLANTEAMIENTO METODOLÓGICO Y PROGRAMACIÓN SEMANAL}

Esta integración de contenidos se realiza tanto a través de los recursos y materiales docentes, como de las técnicas propuestas para trabajarlos dentro y fuera del aula. Los manuales de la asignatura y demás recursos bibliográficos disponibles en la biblioteca de la facultad (libros, revistas, casos, etc.), se combinan con la puesta a disposición de todo tipo de materiales digitales a través de la plataforma Moodle, que se actualizan con periodicidad anual. Todos los materiales -bibliografía, webgrafía, informes sectoriales y otras referencias de grey literaturese incardinan en el tema correspondiente para complementar e ilustrar los contenidos teóricos de la manera más precisa posible. Para aumentar la motivación y el interés del alumno, se utilizan además en el aula recursos tales como software de apoyo visual (e.g. PowerPoint, Prezi), la proyección de vídeos, DVDs y otros soportes multimedia, plataformas interactivas (i.e. Moodle), o artículos o posts de actualidad en páginas web y blogs especializados.

En cuanto a las técnicas docentes, la lección magistral es el principal eje del plan didáctico durante las sesiones teóricas, pues proporciona a los estudiantes una primera visión global de los contenidos y hace hincapié en su conceptualización y relevancia. Se intenta no obstante 
que todas las lecciones magistrales incluyan alguna dinámica participativa, y se utilizan a tal efecto discusiones dirigidas. En las sesiones prácticas se utilizan intensivamente metodologías dirigidas a lograr la participación activa y crítica del alumno. Los estudiantes buscan, seleccionan, analizan y evalúan la información; asumiendo un papel activo en la construcción de su propio conocimiento y enfrentando el reto de comunicarlo de forma efectiva. Fundamentalmente se recurre al aprendizaje basado en problemas, al estudio de casos 0 al role play; utilizando tanto grey literature o noticias de actualidad como recursos publicados por escuelas de negocio de prestigio. ${ }^{i}$ En definitiva, se pretende que los estudiantes se acostumbren a debatir sobre los retos empresariales en público, a diagnosticar las posibles causas de un problema, a identificar soluciones alternativas, y a reflexionar sobre las ventajas e inconvenientes de cada posible curso de acción.

Asimismo, estas metodologías en aula se acompañan de otras actividades realizadas fuera de las horas de clase, tales como la realización de trabajos prácticos individuales o en equipo, el desarrollo de tutorías grupales o individuales, y la planificación (en la medida de lo posible) de seminarios o conferencias a cargo de profesionales externosii.

En resumen, se procura la participación activa de todos los alumnos en todas las sesiones, y su trabajo y colaboración tanto dentro como fuera del aula. Ahora bien, el despliegue del plan didáctico se adapta continuamente según se trate de sesiones teóricas o prácticas, en el aula o fuera de ella, con trabajo individual o grupal (Figura 2). 


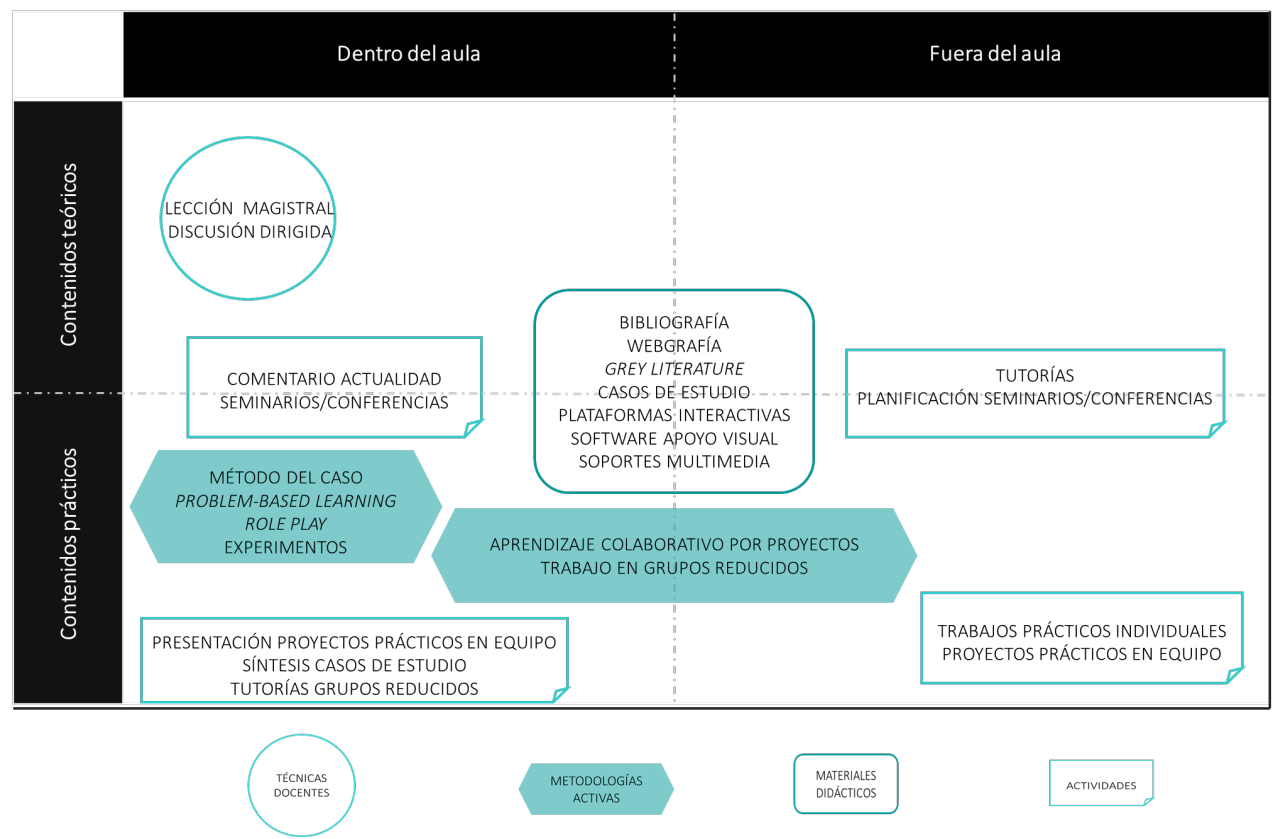

Fuente: elaboración por las autoras.

Figura 2. Diseño del plan didáctico de la materia Distribución Comercial.

El desarrollo cronológico del proceso de enseñanza-aprendizaje se ve facilitado por la programación semanal, una herramienta flexible de planificación docente que calendariza las sesiones y actividades tanto longitudinal (desde las tendencias generales a los retos concretos del punto de venta; desde los fabricantes hasta los consumidores), como transversalmente (favoreciendo el tránsito continuo entre los bloques teóricos y la correspondiente práctica individual o grupal). La programación semanal facilita al profesor responsable de la materia la coordinación de la misma dentro del tiempo disponible, para las numerosas secciones y grupos de alumnos, y con los distintos profesores participantes en su impartición; alineando el proceso de enseñanza-aprendizaje con los objetivos pedagógicos para todas las partes implicadas. Enfatiza además la necesidad de que los alumnos asistan a todas las sesiones de la asignatura por su elevado grado de interdependencia, incluidas las presentaciones de los proyectos prácticos en equipo; siendo todos los contenidos compartidos en el aula 0 a través de la plataforma Moodle evaluables. 


\subsection{INTEGRACIÓN DE METODOLOGÍAS ACTIVAS: APRENDIZAJE COLABORATIVO POR PROYECTOS}

Dentro del espectro de metodologías activas, los proyectos prácticos en equipo -que integran las metodologías de aprendizaje colaborativo y aprendizaje por proyectos- constituyen el eje central de la evaluación continua de la asignatura. Elaborados en grupos reducidos (de 5 integrantes máximo), estos proyectos exigen que los estudiantes profundicen mediante el estudio autónomo sobre problemas, retos y situaciones reales relacionadas con aspectos clave del temario. Dentro del aula, deberán demostrar su dominio de los conceptos teóricos desde una perspectiva práctica, y comunicarlos mediante el empleo pertinente de terminología específica. Para ello, cada equipo trabaja sobre un documento base elegido por el profesor responsable y calendarizado en la programación semanal, que proporciona a los grupos un primer peldaño a partir del cual armar la estructura final del proyecto práctico en equipo.

Los alumnos protagonizan su aprendizaje al dedicarse a: 1) localizar, seleccionar y revisar documentación complementaria que les ayude a entender, actualizar y llevar a la práctica los retos reales planteados en el documento base; y 2) priorizar y extrapolar dichos contenidos, seleccionando y sintetizando las ideas y datos más relevantes sobre la temática asignada, así como ilustrarlos con otros ejemplos reales de problemáticas similares. Cada equipo expone y discute el proyecto resultante con el resto de estudiantes y con el profesor en sesión pública, plenaria e interactiva dentro del aula, en las fechas y horarios calendarizados y difundidos previamente en Moodle. Los equipos se sirven de soportes visuales tipo PowerPoint, Prezi 0 similar, y entregan al profesor una copia física de la presentación antes de cada exposición. Al margen de las recomendaciones o requerimientos formales, el proyecto es fruto del trabajo creativo y la toma de decisiones en el seno del equipo; son los alumnos quienes deciden qué contenido presentar, con qué orden y estructura, bajo qué diseño y estilo, o qué herramientas digitales utilizar. Los contenidos, técnicas y actividades empleados por los estudiantes en el marco de la exposición y presentación de los proyectos prácticos incluyen role play, experimentos en el aula, cata ciega de productos, ambientación de la puesta en escena, así 
como juegos interactivos y producciones multimedia creadas ad hoc. Cada presentación de equipo va seguida de un turno de preguntas del profesor y culmina con un ejercicio de síntesis (wrap-up) donde el docente incardina los aprendizajes específicos dentro de los contenidos correspondientes del plan didáctico, realizando una última iteración del tránsito entre teoría y práctica.

\section{CONCLUSIONES}

La propuesta de criterios programáticos aquí fundamentada e ilustrada puede ser de utilidad para innovar el diseño e implementación de los planes docentes de todas aquellas materias del ámbito de las ciencias de la empresa en las que resulta pertinente combinar distintos niveles de análisis que demandan aproximaciones disciplinarias complementarias (fundamentalmente económicas, pero también sociológicas o jurídicas). También resulta de potencial interés para las materias cuyo objeto de análisis se encuentra en cambio disruptivo bajo presiones del entorno derivadas de la globalización, la revolución digital o el cambio climático. Este puede ser el caso de muchas de las materias adscritas a áreas como comercialización, organización o economía financiera y contabilidad. Las transformaciones socioeconómicas, tecnológicas 0 medioambientales provocan cambios en la posición competitiva y equilibrios de poder de las empresas y demás partes intervinientes en el sector, industria o función en cuestión; plantean problemas prácticos inéditos y demandan respuestas corporativas innovadoras.

Creemos que la continua actualización de contenidos y recursos que exige llevar a la práctica esta propuesta programática, combinada con una aproximación metodológica que insta a los estudiantes a trabajar algunas de las competencias técnicas, interpersonales o sociales más valoradas por las empresas, puede contribuir sin duda a mejorar la empleabilidad de los egresados de ciencias de la empresa. En España los sectores que lideran el crecimiento -los más intensivos en el uso de capital humano altamente cualificado- son precisamente los que presentan una mayor dificultad para cubrir sus vacantes de empleo, debido a la escasez de talentos que aúnen, entre otras competencias, la «capacidad de resolver problemas 
complejos», las «competencias sociales», la «inteligencia práctica» y la capacidad de realizar «trabajo independiente» (Arellano Espinar et al. 2019). Por otro lado, la incorporación de jóvenes titulados que sumen, a sus conocimientos y competencias, la capacidad de comprender retos empresariales de naturaleza sistémica e idear soluciones creativas desde criterios de sostenibilidad -considerando los impactos económicos, sociales y medioambientales de las decisiones corporativas- puede ayudar a las empresas a navegar mejor las aguas turbulentas de la transición necesaria hacia un nuevo contrato social y hacia un modelo productivo basado en la economía circular (Rey-García 2019a).

Por último, el caso de estudio presentado para la materia "Distribución Comercial" ilustra la escalabilidad de las metodologías activas en general, y del aprendizaje colaborativo por proyectos en particular, para motivar al alumnado a que aplique de forma crítica los conocimientos teóricos que está adquiriendo al diagnóstico y solución de problemas empresariales de índole práctica; y al tiempo desarrolle sus competencias de trabajo en equipo y comunicación interpersonal.

\section{REFERENCIAS}

Arellano Espinar, A.; Díez Catalán, L., \& Neut Smith, R.A. (2019). "Revolución tecnológica y desarrollo socioeconómico: una agenda para el mercado de trabajo". En Doménech, R. \& Montes Gan, V. (Eds.): Un nuevo contrato social en una nueva economía. Revista de Economía ICE Información Comercial Española. Noviembre-Diciembre 2019. N. ${ }^{0} 911$. Pp. 79-92.

Barkley, E.F., Cross, K.P. \& Major, C.H. (2012). Técnicas de aprendizaje colaborativo. Madrid: Morata.

Espinosa, J.K., Jiménez, J., Olabe, M. \& Basogain, X. (2006). Innovación docente para el desarrollo de competencias en el EES. VII Congreso de Tecnologías Aplicadas a la Enseñanza de la Electrónica, Madrid. 
Fernández, A. (2006). Metodologías activas para la formación de competencias. Educatio Siglo XXI (24).

García, L. (2014). La Guía Didáctica. Contextos Universitarios Mediados (14).

Rey-García, M. (2011-2019). Guía docente de la materia Distribución Comercial/Retail Management.

Rey-García, M. (2019a). "La empresa en un nuevo contrato social para la sostenibilidad: del principio ético global a los modelos de negocio innovadores". En Doménech, R. \& Montes Gan, V. (Eds.): Un nuevo contrato social en una nueva economía. Revista de Economía ICE Información Comercial Española. Noviembre-Diciembre 2019. N. ${ }^{0} 911$. Pp. 41-52

Rey-García, M. (2019b). Proyecto docente e investigador para el ingreso en el cuerpo de profesores titulares de universidad. Área de Conocimiento de Comercialización e Investigación de Mercados, Departamento de Empresa, Facultad de Economía y Empresa, Universidad de A Coruña. A Coruña Julio 2019.

Sammut-Bonnici, T. \& Galea, D. (2015). PEST analysis. Strategic Management, 12.

\footnotetext{
' Por ejemplo los casos publicados por IESE Business School "Ignacio Valente (A)" y "Eye Care (A), (B), (C) y (D)" y la correspondiente nota técnica sobre el método del caso; o el role play de negociación "El Batmovil".

ii Esta posibilidad se ha materializado durante los últimos cursos en la organización de seminarios impartidos por invitación tanto en el ámbito profesional (p.ej. por el consejero delegado del Grupo Pérez Rumbao, el responsable de relaciones institucionales de Mercadona, o el responsable de sostenibilidad de Leroy Merlin), como en el ámbito académico (p.ej. por la profesora visitante en el marco del Programa Erasmus Marisa Ferreira del Instituto Politécnico do Porto (IPP) que ha impartido una sesión sobre "Innovation and Sustainability" en la distribución comercial en el curso 2018/2019).
} 
